\title{
Electrical properties of InSb $p$-n junctions prepared by diffusion methods
}

\author{
A.V. Sukach ${ }^{1}$, V.V. Tetyorkin ${ }^{1}$, A.I. Tkachuk ${ }^{2}$ \\ ${ }^{1}$ V. Lashkaryov Institute of Semiconductor Physics, NAS of Ukraine, \\ 41, prospect Nauky, 03028 Kyiv, Ukraine \\ ${ }^{2}$ V. Vinnichenko Kirovograd State Pedagogical University, Kirovograd, Ukraine \\ Tel. (38 044) 525-1813, e-mail: teterkin@isp.kiev.ua
}

\begin{abstract}
InSb $p-n$ junctions were prepared by three diffusion methods, including isothermal, two-temperature and two-stage diffusion processes. The current-voltage characteristics were measured as a function of temperature and bias voltage. The highest values of the resistance-area product at zero bias have been obtained for the junctions prepared using the two-stage diffusion process.
\end{abstract}

Keywords: InSb, photodiode, two-stage diffusion, annealing.

Manuscript received 12.04.16; revised version received 20.07.16; accepted for publication 13.09.16; published online 04.10.16.

\section{Introduction}

InSb and $\mathrm{HgCdTe}$ photodiodes are basic photodetectors for effective registration of IR radiation in the spectral range of 3 to $5 \mu \mathrm{m}$ [1]. Both photodiodes have comparable operational characteristics and threshold parameters at $77 \mathrm{~K}$. Widespread usage of $\mathrm{InSb}$ photodiodes is based on well developed technology of bulk material, long-term stability and reproducibily of their parameters. Up to date, $2052 \times 2052$ focal matrix is developed on InSb bulk material [2]. The commonly used methods for manufacture InSb $p$ - $n$ junctions are implantation of light ions $\mathrm{Be}$ [3-5], $\mathrm{Mg}$ [3] and $\mathrm{Zn} \mathrm{[6],} \mathrm{as}$ well as diffusion of acceptor impurity $\mathrm{Cd}$ [7-12]. In diffusion photodiodes, rather high value of the differential resistance - area product $R_{0} A=$ $3.4 \cdot 10^{3} \Omega \cdot \mathrm{cm}^{2}$ was already reported in 1961 [7]. For comparison, in modern commercial photodiodes this parameter has typical values of $5 \cdot 10^{3} \Omega \cdot \mathrm{cm}^{2}$ [13]. Moreover, the specific detectivity in commercial photodiodes $D_{\lambda}^{*}=1 \cdot 10^{11} \mathrm{~cm} \cdot \mathrm{Hz}^{1 / 2} \cdot \mathrm{W}^{-1}$ is almost five times less than the theoretically possible value [13]. The reason for this discrepancy may be excessive currents, which nature is not fully understood yet. A model for the trap-assisted tunnelling current flowing through the depletion region has been developed for interpretation of experimental data in $\mathrm{HgCdTe}$ and InSb photodiodes [1]. This model involves uniform distribution of deep traps through the depletion region $[8,11]$. On the contrary, in a model of non-homogeneous $p-n$ junction, carrier tunnelling with participation of extended defects, such as dislocations, is assumed to be responsible for the excess current $[12,14]$. In this case, local areas exist in the depletion region, which are characterized by higher electric field. The dark current can flow through these areas by means of trap-assisted or interband tunnelling as well as through the uniform regions by means of generation-recombination or diffusion mechanism [15]. Obviously, grown-in and process-induced dislocations can effect on the carrier transport. The process-induced 
dislocations in InSb photodiodes are related with high plasticity of bulk material. High concentration of point and extended defects in freshly prepared junctions results in a problem of separation of electrical and metallurgical junctions. In implanted photodiodes, this problem is resolved by photonic or thermal annealing [4], whereas in diffusion ones two-temperature diffusion method is used $[9,12,14]$.

Based on the above mentioned, the purpose of this study is to clarify impact of various diffusion methods on dark current and threshold parameters of InSb $p-n$ junctions.

\section{Samples and experimental methods}

The investigated $p-n$ junctions were produced by diffusion of cadmium into single crystal substrates of $n$ type conductivity with crystallographic orientation (100) and average thickness close to $500 \mu \mathrm{m}$. The concentration and mobility of electrons in the substrates were $(1 \ldots 2) \cdot 10^{15} \mathrm{~cm}^{-3}$ and $5.0 \cdot 10^{5} \mathrm{~cm}^{2} / \mathrm{V} \cdot \mathrm{s}$ at $77 \mathrm{~K}$, respectively. The average density of dislocations (etch pits density) was less than $350 \mathrm{~cm}^{-2}$ [16]. The damaged layer was removed by chemical dynamic polishing using a polishing etchant $2 \% \mathrm{Br}_{2}+\mathrm{HBr}$. The surface quality was controlled by an interference microscope. The measured roughness at the surface after polishing was $\sim 0.03 \mu \mathrm{m}$. To prevent re-evaporation of constituents, additionial amount of $\mathrm{InSb}$ and elemental $\mathrm{Sb}$ was placed into a silica ampoule to ensure the saturation vapor condition.

Three methods of cadmium diffusion into $n$-InSb substrates were implemented: i) isothermal diffusion at $420{ }^{\circ} \mathrm{C}$ for $30 \mathrm{~min}$; ii) two-temperature diffusion for $30 \mathrm{~min}$, when $\mathrm{Cd}$ source and $\mathrm{InSb}$ substrate were in different temperature zones 380 and $420^{\circ} \mathrm{C}$; iii) twostage diffusion, when after isothermal diffusion of $\mathrm{Cd}$ at $380{ }^{\circ} \mathrm{C}$ the thermal annealing at $420^{\circ} \mathrm{C}$ was carried out for $60 \mathrm{~min}$ in a separate ampoule. Hereafter, the $p$ - $n$ junctions prepared by these methods are labeled as TR1, TR2 and TR3, respectively.

The $p-n$ junction depth was determined by measuring the sign of thermo-EMF by using the probe method during sequential chemical etching the doped surface layer. The determined values were $3.5 \pm 0.5$, $3.0 \pm 0.5$ and $4.5 \pm 0.5 \mu \mathrm{m}$ for TR1, TR2 and TR3 junctions, respectively. The active area of mesa structures was $1.4 \cdot 10^{-2} \mathrm{~cm}^{2}$. As determined by the differential Hall effect [17], the average concentration of holes in the doped layer was $(7 \pm 1) \cdot 10^{18} \mathrm{~cm}^{-3}$ at $77 \mathrm{~K}$. Ohmic contacts to $p$ - and $n$-type regions of the junctions were prepared using In-Zn alloy and pure In, respectively. Formation of ohmic contacts and purification of mesas was carried out in a hydrogen atmosphere at $\sim 350{ }^{\circ} \mathrm{C}$ for 5 to $10 \mathrm{~min}$. Thin polycrystalline films of $\mathrm{CdTe}$ were used as passivation and protective layers due to good agreement between lattice parameters and thermal expansion coefficients of $\mathrm{CdTe}$ and InSb. By using polycrystalline CdTe, the density of interface states in the CdTe-InSb heterojunction can be 3 to 4 times reduced as compared to that at the oxide-InSb interface [18]. The currentvoltage and high-frequency $(1 \mathrm{MHz})$ capacitancevoltage characteristics were measured as functions of the bias voltage and temperature.

\section{Results and discussion}

The capacitance-voltage characteristics are shown in Fig. 1. As seen, experimental data are linearized in coordinates $C^{-3}-U$, which indicates formation of linearly-graded $p-n$ junctions. The dopant concentration gradient $a$, as well as the diffusion voltage $U_{D}$ and the depletion layer width at zero bias $W_{0}$ have been estimated from the capacitance measurements. Appropriate data are summarized in Table. Note that the diffusion voltage equals $180 \mathrm{mV}$ for TR1 junction and $160 \mathrm{mV}$ for TR2 and TR3 junctions.

Typical current-voltage characteristics are shown in Fig. 2. The following peculiarities should be pointed out. In TR1 and TR2 junctions, the forward current exhibits two exponential branches, which can be approximated by the following expression:

$$
I=I_{01} \exp \left[\frac{e\left(U-I R_{S}\right)}{E_{0}}\right]+I_{02} \exp \left[\frac{e\left(U-I R_{S}\right)}{\beta k T}\right],
$$

where the characteristic energy $E_{0}$ equals 29 and $32 \mathrm{meV}$ for TR1 and TR2 junctions, respectively. Other parameters are shown in Table. At the same time, in the TR3 junction only one exponential branch is observed, which is described by the second term in the equation (1).

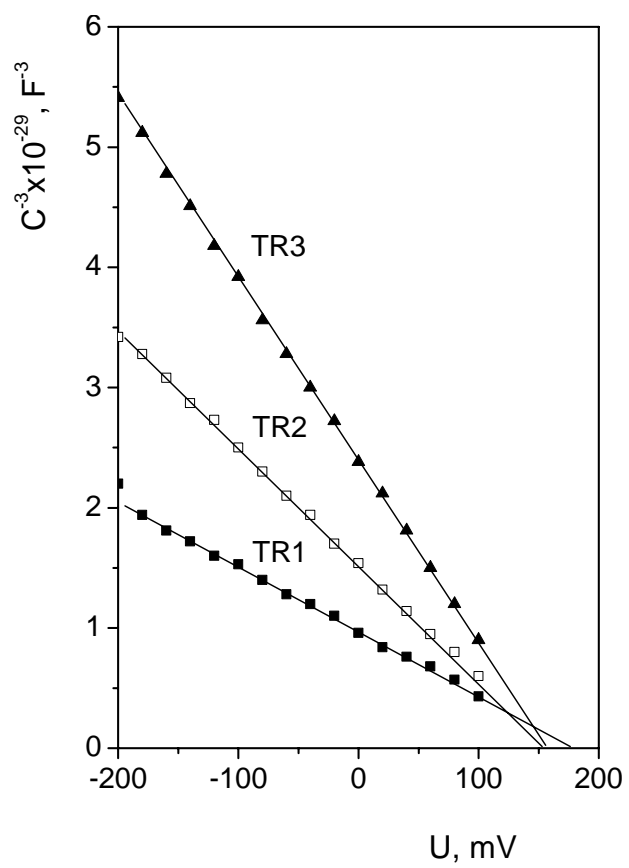

Fig. 1. Capacitance-voltage characteristics of InSn $p-n$ junctions prepared using TR1, TR2, TR3 methods of diffusion. 


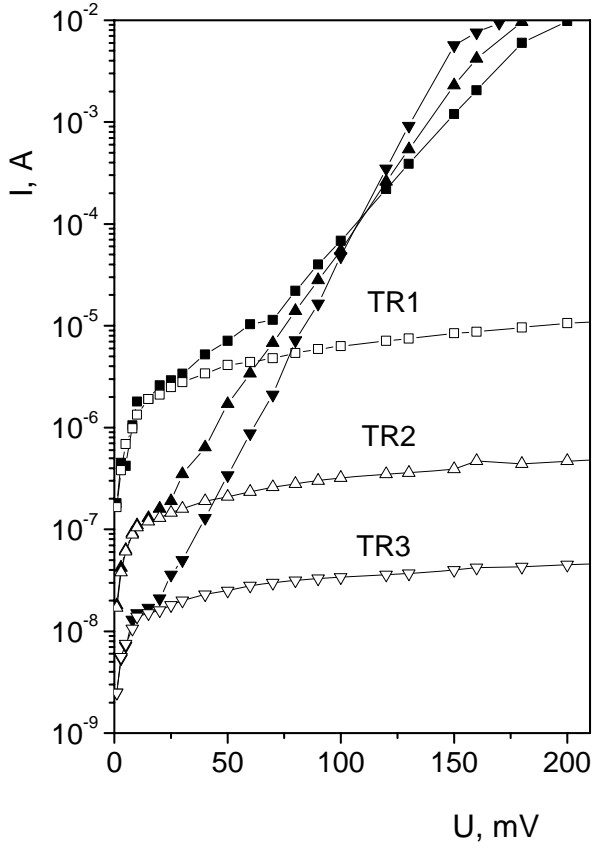

Fig. 2. Forward (open dots) and reverse (close dots) currentvoltage characteristics of InSb $p$ - $n$ junctions.

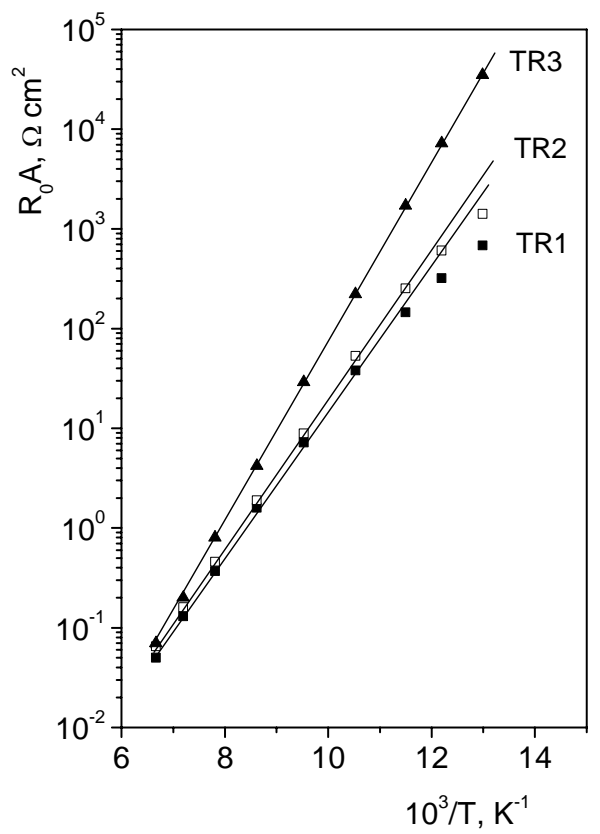

Fig. 3. Temperature dependences of the differential resistancearea product.

The temperature dependences of $R_{0} A$ product is shown in Fig. 3. The differential resistance was obtained from the forward $I-U$ characteristics at the bias voltage $U=10 \mathrm{mV}$. In the temperature range $87-150 \mathrm{~K}$, the experimental data are well linearized in the coordinates $\ln \left(R_{0} A\right)-10^{3} / T$. At temperatures $\leq 87 \mathrm{~K}$, deviation from the linear dependence is observed for TR1 and TR2 junctions, which is caused by the influence of the tunnel current. The tunnel current is also observed at low forward biases in these junctions (see Fig. 2, curves 1 and 2). A

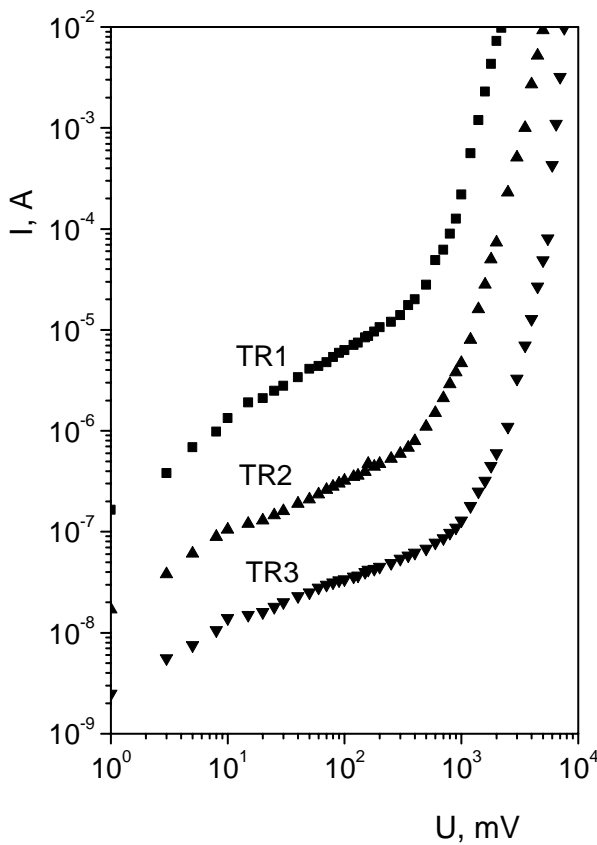

Fig. 4. Reverse current-voltage characteristics of InSb $p-n$ junctions.

distinctive feature of the current-voltage characteristic of TR3 junction is absence of tunnel current component, which is also confirmed by a linear dependence of $R_{0} A$ on the reverse temperature in the whole temperature range $77 \ldots 150 \mathrm{~K}$. The activation energy for a differential resistance is estimated to be $180 \mathrm{meV}$ for TR3 junction. Taking into account the ideality coefficient in this junction $\beta=1.6$, one can conclude about the contribution of diffusion and generation-recombination currents to the total dark current in a roughly equal proportion. Lower activation energy of $140 \mathrm{meV}$ and higher value of the ideality coefficient for TR1 and TR2 junctions is explained by the main contribution of generationrecombination current to the measured dark current. Note that the value of the product $R_{0} A=3.5 \cdot 10^{4} \Omega \cdot \mathrm{cm}^{2}$ at 77 for TR3 junction almost an order of magnitude higher than that of commercial photodiodes [13]. It can be explained by passivation of mezas with cadmium telluride as well as the higher quality of the junction itself. The detailed analysis of tunnel currents in diffusion InSb junctions is given in $[12,14]$. An additional evidence of the TR3 junction quality was obtained from the analysis of generation current at the reverse biases. The effective lifetime $\tau_{0}$ in the depletion region is almost an order of magnitude higher in comparison with TR1 and TR2 junctions (see Table).

The reverse current-voltage characteristics are shown in Fig. 4. The experimental data were approximated by a power dependence $I \sim U^{m}$. The exponent $m$ equals 1.0 and 0.6 in TR1 and TR2 for the voltage ranges $1 \ldots 10$ and $10 \ldots 200 \mathrm{mV}$, respectively. The appropriate value of 0.4 was obtained for TR3 junction. At the reverse biases $\geq 1 \mathrm{~V}$, the exponent $m$ sharply increases to 5 for TR1 and TR2 junctions, and 
up to 13 for TR3 junction. The breakdown voltage $U_{B}$ that is measured at the current density $1.0 \mathrm{~A} / \mathrm{cm}^{2}$ [19] was 2.3, 5.4 and 7.5 V for TR1, TR2 and TR3 junctions, respectively. Experimental values of $m$ and $U_{B}$ for TR1 and TR2 junctions can be explained within a model of tunneling breakdown in the inhomogeneous $p-n$ junction [14]. A sharp increase in the current in TR3 junction and higher values of the breakdown voltage can be explained by the avalanche breakdown [9]. However, additional investigations are required for it. It should be pointed out that in TR3 junction the dark current density at the reverse bias $0.2 \mathrm{~V}$ equals to $3.2 \cdot 10^{-6} \mathrm{~A} / \mathrm{cm}^{2}$, which is several orders of magnitude less than the values reported in literature [3, 6, 7, 14]. However, this value exceeds almost three times the theoretical one required to implement BLIP regime in InSb photodiodes [4]. An additional reason for the large dark currents in TR1 and TR2 junctions in comparison with TR3 junctions may be generation of point and extended defects at the metallurgical junction caused by retrograde solubility of cadmium in InSb [20].

Table. Parameters of diffusion InSb $p-n$ junctions at $T=77 \mathrm{~K}$.

\begin{tabular}{|c|c|c|c|c|c|c|c|}
\hline 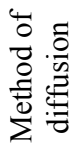 & $\begin{array}{l}\ll \\
\stackrel{\overrightarrow{0}}{0}\end{array}$ & 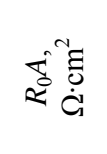 & $\begin{array}{l}\varangle \\
\stackrel{\tilde{S}}{-1}\end{array}$ & $\infty$ & $\begin{array}{l}{ }^{\dagger} \\
\Xi \\
0 \\
0 \\
0\end{array}$ & $\frac{\Xi}{3}$ & $\begin{array}{l}n \\
\hat{\beta}\end{array}$ \\
\hline TR1 & $1.3 \cdot 10^{-6}$ & $6.8 \cdot 10^{2}$ & $2.2 \cdot 10^{-7}$ & 2.7 & $2.3 \cdot 10^{19}$ & 1.0 & $1.6 \cdot 10^{-9}$ \\
\hline TR2 & $8.4 \cdot 10^{-8}$ & $1.4 \cdot 10^{3}$ & $5.0 \cdot 10^{-8}$ & 2.1 & $1.3 \cdot 10^{19}$ & 1.1 & $7.9 \cdot 10^{-9}$ \\
\hline TR3 & - & $3.5 \cdot 10^{4}$ & $4.0 \cdot 10^{-9}$ & 1.6 & $8.5 \cdot 10^{18}$ & 1.3 & $1.2 \cdot 10^{-7}$ \\
\hline
\end{tabular}

\section{Conclusions}

The lowest dark current $3.2 \cdot 10^{-6} \mathrm{~A} / \mathrm{cm}^{2}$ at the reverse bias $0.2 \mathrm{~V}$ was obtained in $p-n$ junctions produced by two-stage diffusion of cadmium in $n$-InSb (100) substrates at $380^{\circ} \mathrm{C}$ for $30 \mathrm{~min}$ followed by thermal annealing at $420^{\circ} \mathrm{C}$ for $60 \mathrm{~min}$. In the temperature range $77-150 \mathrm{~K}$ the differential resistance-area product at zero bias is determined by the dark current composed of diffusion and generation-recombination components with approximately the same partial contribution.

\section{References}

1. A. Rogalskii (Ed.), Infrared Photon Detectors. SPIE Optical Engineering Press, 1995.

2. A. Rogalski, Optical detectors for focal plane arrays // Opto-Electron. Rev. 12 (2), p. 221-245 (2004).

3. E. Hurwitz, I.P. Donnely, Planar InSb photodiodes fabricated by Be and $\mathrm{Mg}$ implantation // Solid State Electron. 18(9), p. 753-756 (1975).

4. V.P. Astahov, D.A. Gindin, V.V. Karpov et al., Developments in InSb-photodetectors with verylow-level dark current for use in high performance
IR CCDs // Prikladnaya Fizika, 2, p. 73-79 (1999), in Russian.

5. V.P. Astahov, D.A. Gindin, V.V. Karpov, A.V. Talimov, On the possibility of increasing the current sensitivity in InSb-based photodiodes // Prikladnaya Fizika 1, p. 56-62 (2002), in Russian.

6. P.V. Birulin, V.I. Turinov, E.B. Yakimov, Characteristics of InSb photodiode linear arrays // Semiconductors (Springer), 38(4), p. 488-503 (2004).

7. H.J. Stocker, Current-voltage characteristics of alloyed and diffused $p-n$ junction diodes in $\mathrm{InSb} / /$ J. Appl. Phys. 32(2), p. 322 (1961).

8. R. Adar, V. Nemirovsky and I. Kidron, Bulk tunneling contribution to the reverse breakdown characteristics of $\mathrm{InSb}$ gate controlled diodes // Solid State Electron. 30(12), p. 1289-1293 (1987).

9. S.L. Tu, K.F. Huang and S.J. Yang, InSb p-n function with avalanche breakdown behavior // Jpn. J. Appl. Phys. 28(11), p. L1874-L1876 (1989).

10. A.M. Filachev, I.D. Burlakov, A.I. Dirochka et al., Fast-operating array photodetective assembly of a $128 \times 128$ elements format on the basis of InSb with the frame-accurate accumulation and function of the range finder // Prikladnaya Fizika, 2, p. 21-25 (2005), in Russian.

11. M. Moradi, M. Darace, M. Hajian et al., Optimum concentration of InSb photodiode for minimum low reverse bias leakage current // Ukr. J. Phys. 55(4), p. 422-424 (2010).

12. A. Sukach, V. Tetyorkin, A. Voroschenko, A. Tkachuk et al., Carrier transport mechanisms in InSb diffusion p-n junctions // Semiconductor Physics, Quantum Electronics and Optoelectronics, 17(4), p. 325-330 (2014).

13. Indium Antimonide Detectors. Catalog. Teledyne Judson Technologies, 2015.

14. A.V. Sukach, V.V. Tetyorkin, A.I. Tkachuk, Carrier transport mechanisms in reverse biased InSb p-n junctions // Semiconductor Physics, Quantum Electronics and Optoelectronics, 18(3), p. 267-271 (2015).

15. V. Tetyorkin, A. Sukach and A. Tkachuk, InAs infrared photodiodes // Advances in Photodiode. Ed. Gian-Franco Dalla Betta, INTECH, p. 427-446, 2011.

16. F. Dewald, The kinetics and mechanism of formation of anode films on single crystal InSb // J. Electrochem. Soc. 104(4), p. 244-251 (1957).

17. M. Schröder, Semiconductor Materials and Devise Characterization. Wiley, 2006.

18. Yu.F. Bikovskii, L.A. Vjukov, A.G. Dudoladov et al., Investigation of MIS film structures based on CdTe-InSb // Pisma Zhurnal Tekhn. Fiziki, 9(17), p. 1071-1074 (1983), in Russian.

19. S.M. Sze, Physics of Semiconductors Devices. Second Edition, Wiley, 1981.

20. B.I. Boltaks, V.I. Sokolov, Investigation of cadmium diffusion in indium antimonide by layer autoradiography method // Fizika Tverdogo Tela, 5(4), p. 1077-1081 (1963), in Russian. 